\title{
Electrogastrogram Analysis in Children with Chronic Abdominal Pain
}

\author{
Li Ping Yuan*, Cai Jie, Qi-Lian Zhou and Gui Ming \\ Department of Pediatrics, First Affiliated Hospital of Anhui Medical University, Hefei, China
}

${ }^{*}$ Corresponding author: Li Ping Yuan, Department of Pediatrics, The First Affiliated Hospital of Anhui Medical University, Hefei, China, Tel: (+ 86)-5516292-2058; E-mail: yuanliping3986@sina.com

Received: 11 Mar, 2019 | Accepted: 27 Mar, 2019 | Published: 01 Apr, 2019

Citation: Yuan LP, Jie C, Zhou QL, Ming G (2019) Electrogastrogram Analysis in Children with Chronic Abdominal Pain. J Clin Lab Med 4(1): dx.doi.org/10.16966/2572-9578.125

Copyright: (C) 2019 Yuan LP, et al. This is an open-access article distributed under the terms of the Creative Commons Attribution License, which permits unrestricted use, distribution, and reproduction in any medium, provided the original author and source are credited.

\section{Abstract}

Objective: Electrogastrography (EGG) is a non-invasive method for the diagnosis of gastric electrical activity. This study used an EGG to analyze the gastric activity in children with Chronic Abdominal Pain (CAP).

Methods: We conducted a retrospective analysis of patients with CAP who underwent EGG at the first affiliated Hospital of Anhui Medical University. Multichannel EGG with 8-minutes recordings preprandially and postprandially was done in children with CAP. Computerized spectral analysis methods were used to compute various EGG parameters.

Results: Chronic superficial gastritis and Functional Dyspepsia (FD) were very common among 96 patients with CAP. The dominant power and waveform response area of EGG was significantly lower in FD patients and chronic superficial gastritis in both preprandial and postprandial states. DP, WRA and power ratio was obviously higher in patients with superficial gastritis, duodenitis and reflux esophagitis in their postprandial states.

Conclusions: Gastric myoelectrical activity abnormalities were observed in CAP patients and different abnormality of EGG parameters was shown in different groups. EGG is an effective, reliable and non-invasive method in the diagnosis of CAP subgroups.

Keywords: Chronic abdominal pain (CAP); Electrogastrogram (EGG); Gastritis; Functional dyspepsia

\section{Background}

Chronic Abdominal Pain (CAP) is a group of symptoms for which children and their parents seek doctors' attention and diagnosis. It is a surprisingly common problem in pediatric primary and specialty care [1]. Early diagnosis of abdominal pain predominantly relied on laboratory tests, abdominal ultrasound scan, gastrointestinal endoscopy, and gastrointestinal contrast and so on. However, most of the children couldn't receive these examinations due to their younger age or their parents' warning about some invasive examinations [2]. Electrogastrography (EEG) is a technique used to record the gastric smooth muscle electrical activity. It is commonly applied in children with Functional Dyspepsia (FD), abdominal pain, chronic intractable nausea, vomiting, and gastroparesis because EGG is more easily applicable than the other diagnostic techniques and is in a noninvasive fashion $[3,4]$. The aim of this study was to analyze the gastric myoelectrical activity in children with CAP.

\section{Research Design}

\section{Participants}

A retrospective study was conducted on 120 inpatients and outpatients (aged 3-15 years old) with continuous or intermittent mid-upper abdominal pain for at least three months coming to the First Affiliated Hospital, Anhui Medical University between March
2017 and December 2018. The patients underwent urine and stool routine test, EGG, abdominal ultrasound scan, abdominal X-ray, upper gastrointestinal endoscopy or gastrointestinal contrast at our department. Some patients were excluded because they had an intestinal obstruction, intestinal dilatation, intussusceptions, Hirschsprung's disease.

Gastric myoelectrical activity was recorded by an EGG for which 4 locations (channels) on the thoraco-abdominal skin surface. An EGEG-2D8-type double-lead smart gastrointestinal electrogastrogram instrument (Institute of Electric Technology Kelly Optoelectronics Technology Co., Ltd, Hefei, China) which is an FDA-approved clinical instrument was used. 4 active electrodes were placed above the stomach as previously described. After an overnight fast, the EEG recording was made in each subject for 8 minutes in the fasting state and the fed state (a 5-min break after a standardized soft diet that consists of milk (200 ml) and bread $(100 \mathrm{~g}), 1300$ and $2850 \mathrm{~kJ}$, respectively. We measured and carried out analyses of the following parameters during the preprandial and postprandial periods: 1) Dominant Power (DP) $(\mu \mathrm{V})$; 2) Dominant Frequency (DF) (CPM); 3) PERCENT OF NORMAL SLOW WAVE (PNSW); 4) Waveform Response Area (WRA).

The power ratio is used as an indicator of changes in gastric contractility. It is generally accepted that a power ratio $>1$ reflects 
an increase in gastric contractility after the intervention, whereas a power ratio $<1$ reflects a decrease in gastric contractility. The reference parameters of EGG about diagnostic criteria according to EGG Academic Conference convened by the Chinese Medical Association (Xiamen, 1999) and classification of the EGG system of FDA (USA) [5].

\section{Statistical analysis}

All data were presented as means \pm SD (standard deviation). Student t-test was used for age, DP, DF, WRA, power ratio parameters, and the Pearson chi-square test $\left(\chi^{2}\right)$ test was used for sex analysis. Statistical significance was assigned for $\mathrm{P}<0.05$.

\section{Results}

\section{The demographic characteristics of the patients with CAP}

Among 120 patients, 96 patients were enrolled in our study. They were diagnosed as chronic superficial gastritis $(n=23)$, superficial gastritis $(n=13)$, duodenitis $(n=14)$, reflux esophagitis $(n=16)$, bile reflux gastritis $(n=3)$, gastric ulcer $(n=4)$ and functional dyspepsia $(\mathrm{n}=23)$ via gastrointestinal endoscopy or gastrointestinal contrast. The age was more young among children with duodenitis than those in normal children $(6.64 \pm 1.98$ vs $8.80 \pm 2.53$ years, $\mathrm{p}<0.05)$. The age was old in children with chronic superficial gastritis and functional dyspepsia; however, there was no statistical difference between these two groups and the normal group. And there was no statistical difference between sexes among these groups (Table 1).

\section{Gastric motility evaluation of patients with chronic abdominal pain}

The raw signal and spectral analyses of the EGG signals for CAP patients are illustrated in figure 1 and tables 2-5. The raw signal in chronic superficial gastritis patients showed lower Dominant Power (DP) and a higher incidence of arrhythmia and Brady gastric, which were obviously seen in channel 1 and 4 . And the raw signal in reflux esophagitis and superficial gastritis showed higher DP, the incidence of arrhythmia and tachygastric. The raw signal of duodenitis displayed higher DF and DP. The spectral analyses of the EGG signals revealed that the preprandial DP in chronic superficial gastritis patients it was the lowest among all these patients in our study, and the postprandial DF, WRA and power ratio was significantly reduced. Most of the postprandial parameters (DP, DF, WRA, PNSW and power ratio) in FD patients were the lower, especially power ratio and WRA was the lowest in all the patients with CAP. The postprandial DP, DF, and WRA in patients with reflux esophagitis, duodenitis, and superficial gastritis were higher compared with other patients with CAP and the postprandial DF in patients with reflux esophagitis was the highest among all the patients in channel I.

Table 1: Demographic characteristics of the patients with CAP.

\begin{tabular}{|l|c|c|c|}
\hline \multicolumn{1}{|c|}{ Groups } & $\mathbf{n}$ & Age(years) & Sex(boys/girls) \\
\hline Duodenitis & 14 & $6.64 \pm 1.98^{*}$ & $6 / 8$ \\
\hline Superficial gastritis & 13 & $8.69 \pm 2.46$ & $6 / 7$ \\
\hline Chronic superficial gastritis & 23 & $9.26 \pm 3.22$ & $14 / 9$ \\
\hline Reflux esophagitis & 16 & $8.28 \pm 3.15$ & $8 / 8$ \\
\hline Functional dyspepsia & 23 & $9.09 \pm 3.25$ & $11 / 12$ \\
\hline Normal & 10 & $8.80 \pm 2.53$ & $3 / 7$ \\
\hline
\end{tabular}

Data were expressed as mean $\pm S D, * P<0.05$, compared with normal group

\section{Discussion and Conclusion}

EGG is a noninvasive method for recording the rhythm of gastric electromyogram activity by abdominal surface electrodes. Clinical studies have found that inflammatory infiltration and deformed hyperplasia of gastric mucosa and the muscular layer can cause the generation and transmission of basic electric rhythm, and then lead to abnormal rhythm and ectopic rhythm [6]. A large number of clinical studies have shown that gastrointestinal symptoms are related to gastric motility disorder and abnormal gastric electrical activity. Moreover, the changes of EGG parameters dominant power, frequency and percent of the normal slow wave can reflect gastric motor function [7]. Patients with tachygastria and high DP may have obvious abdominal pain, acid regurgitation, and belching, and patients with bradygastria and low DP may have the upper abdominal fullness, anorexia, fatigue, and emaciation. Waveform Response Area (WRA) and power ratio represent the diastolic and contractile movements of the stomach.

Our present study showed that gastritis, reflux esophagitis, and duodenitis are the main causes of chronic abdominal pain in children, especially chronic superficial gastritis and functional dyspepsia. According to the relevant investigation, chronic superficial gastritis in children is mostly caused by functional dyspepsia and changes in gastric motility $[8,9]$. Uścinowicz $M$, et al. [10] investigated electrogastrography in children with functional abdominal pain and gastritis and found that abnormalities in gastric rhythm were noticed in the highest percent in gastritis in fasting state. Normogastry was dominant in the postprandial period in functional abdominal pain (FAP) and gastritis.

Statistical differences of dominant frequency, dominant power was found among FAP, gastritis, and control. And the higher percent of bradygastry in fasting state compared with a postprandial period was observed in FAP and gastritis [10]. And this study verified much lower EGG parameters DP, WRA, and power ratio in patients with FD and chronic superficial gastritis compared with normal healthy children. These results suggested that the EGG is sensitive and accurate in the diagnosis of chronic superficial gastritis and functional dyspepsia.

Reflux esophagitis is closely related to gastric motility disorders such as delayed gastric emptying, weakened gastric antrum movement and reduced pyloric pressure. Delayed gastric emptying and dysfunction of the pyloric sphincter cause the contents of the stomach and duodenum to flow back into the esophagus and cause inflammation of the stomach and esophageal mucosa, which may affect the electrical activity of the stomach. Its raw signal of EGG showed high frequency, amplitude zigzag wave and spectral analyses of the EGG signals in patients with reflux esophagitis showed high DP, DF and increased PNSW. These EGG wave changes were also reported by Guo et al. [7]. The patients with duodenitis had a high-frequency wave in EGG raw signal and their spectral analyses revealed high DP, DF, and increased power ratio.

As a conclusion, EGG examination in children can help us analyze gastrointestinal electrical activity and abnormal patterns, and provide good research and a diagnostic basis for chronic abdominal pain caused by common digestive diseases such as gastrointestinal motility disorder and functional dyspepsia. However, there is much interference in the electrogastrogram examination in children, and there are still some limitations. So we need larger and larger samples to summarize the clinical experience of EGG and make it implement in clinical practice as widely as possible. 
Table 2: DP, DF, PNSW, WRA and power ratio of EGG (Channel I) in patients.

\begin{tabular}{|c|c|c|c|c|c|}
\hline \multirow[t]{2}{*}{ Groups } & \multirow[t]{2}{*}{$\mathbf{n}$} & \multicolumn{2}{|c|}{$\mathrm{DP}(\mu \mathrm{V})$} & \multicolumn{2}{|c|}{$\mathrm{DF}(\mathrm{cpm})$} \\
\hline & & Preprandial & Postprandial & Preprandial & Postprandial \\
\hline Duodenitis & 14 & $191.79 \pm 90.31$ & $361.57 \pm 96.10^{* * \star \star}$ & $3.13 \pm 0.31$ & $3.35 \pm 0.30^{\star}$ \\
\hline Superficial gastritis & 13 & $231.15 \pm 133.19$ & $332.46 \pm 93.42^{*}$ & $3.25 \pm 0.33$ & $3.28 \pm 0.33$ \\
\hline Chronic superficial gastritis & 23 & $165.65 \pm 73.31$ & $249.17 \pm 117.25^{\# \Delta \Delta}$ & $3.19 \pm 0.25$ & $3.15 \pm 0.27^{\Delta}$ \\
\hline Reflux esophagitis & 16 & $156.19 \pm 52.95 \#$ & $284.81 \pm 73.71^{* *}$ & $3.45 \pm 0.33^{* \star \star \Delta}$ & $3.24 \pm 0.26$ \\
\hline Functional dyspepsia & 23 & $195.70 \pm 74.38$ & $208.21 \pm 96.54^{\# \# \Delta \Delta}$ & $3.33 \pm 0.32$ & $3.13 \pm 0.32^{\Delta}$ \\
\hline Normal & 10 & $184.7 \pm 41.77$ & $250.4 \pm 44.03$ & $3.16 \pm 0.23$ & $3.26 \pm 0.34$ \\
\hline
\end{tabular}

Note: DP=Dominant Power; DF=Dominant Frequency; PNSW=Percent of Normal Slow Wave; WRA=Waveform Response Area

Table 2: (Continue)

\begin{tabular}{|c|c|c|c|c|c|c|}
\hline \multirow{2}{*}{ Groups } & \multirow{2}{*}{$\mathbf{n}$} & \multicolumn{2}{|c|}{ PNSW (\%) } & \multicolumn{2}{|c|}{ WRA } & \multirow{2}{*}{ Power ratio } \\
\hline & & Preprandial & Postprandial & Preprandial & Postprandial & \\
\hline Duodenitis & 14 & $62.29 \pm 7.04$ & $64.51 \pm 12.86$ & $62.29 \pm 7.04$ & $129 \pm 31.59^{* *} \ldots \star \star$ & $3.3 \pm 1.78^{* *}=$ \\
\hline Superficial gastritis & 13 & $63.42 \pm 17.25$ & $64.36 \pm 9.31$ & $63.42 \pm 17.25$ & $120.54 \pm 34.18^{*}$ & $2.32 \pm 1.88$ \\
\hline Chronic superficial gastritis & 23 & $64.40 \pm 10.03$ & $64.8 \pm 7.65$ & $64.40 \pm 10.03$ & $86.96 \pm 41.51^{\#}$ & $2.03 \pm 1.40$ \\
\hline Reflux esophagitis & 16 & $58.14 \pm 7.84^{\star}$ & $63.9 \pm 8.42$ & $58.31 \pm 17.76$ & $98.75 \pm 22.97$ & $1.93 \pm 0.76=$ \\
\hline Functional dyspepsia & 23 & $61.55 \pm 9.80$ & $65.98 \pm 8.05$ & $61.55 \pm 9.80$ & $71.46 \pm 32.21^{\# \# \Delta \Delta . .}$ & $1.17 \pm 0.76^{\# \Delta \Delta \star}$ \\
\hline Normal & 10 & $62.82 \pm 8.80$ & $60.86 \pm 7.37$ & $64.7 \pm 12.07$ & $93.3 \pm 17.44$ & $1.55 \pm 0.52$ \\
\hline
\end{tabular}

${ }^{*} \mathrm{P}<0.05,{ }^{* *} \mathrm{P}<0.01$ compared with normal group; ${ }^{\#} \mathrm{P}<0.05,{ }^{\# \#} \mathrm{P}<0.01$ compared with superficial gastritis group; ${ }^{\Delta} \mathrm{P}<0.05,{ }^{\Delta \Delta} \mathrm{P}<0.01$ compared with duodentis group; $\cdot \mathrm{P}<0.05$ compared with functional dyspepsia group; ${ }^{\star} \mathrm{P}<0.05,{ }^{\star} \mathrm{P}^{\circ}<0.01$ compared with chronic superficial gastritis group

Table 3: DP, DF, PNSW, WRA and power ratio of EGG (Channel 4) in patients.

\begin{tabular}{|c|c|c|c|c|c|}
\hline \multirow{2}{*}{ Groups } & \multirow{2}{*}{$\mathbf{n}$} & \multicolumn{2}{|c|}{$\mathrm{DP}(\mu \mathrm{V})$} & \multicolumn{2}{|c|}{$\mathrm{DF}(\mathrm{cpm})$} \\
\hline & & Preprandial & Postprandial & Preprandial & Postprandial \\
\hline Duodenitis & 14 & $176.57 \pm 70.74$ & $423.5 \pm 108.64^{* *}$ & $3.21 \pm 0.38$ & $3.31 \pm 0.44$ \\
\hline Superficial gastritis & 13 & $162.69 \pm 45.88$ & $408.08 \pm 123.20^{* *}$ & $3.40 \pm 0.44$ & $3.22 \pm 0.29$ \\
\hline Chronic superficial gastritis & 23 & $129.26 \pm 69.41$ & $308.13 \pm 178.08$ & $3.19 \pm 0.30$ & $3.21 \pm 0.29$ \\
\hline Reflux esophagitis & 16 & $169.88 \pm 53.39$ & $420.06 \pm 141.23^{* *} \cdot-$ & $3.34 \pm 0.25$ & $3.25 \pm 0.25$ \\
\hline Functional dyspepsia & 23 & $180.57 \pm 85.48^{\star}$ & $275.58 \pm 162.44$ & $3.4 \pm 0.26$ & $3.21 \pm 0.19$ \\
\hline Normal & 10 & $156.8 \pm 26.84$ & $278.5 \pm 61.42$ & $3.22 \pm 0.20$ & $3.21 \pm 0.20$ \\
\hline
\end{tabular}

Table 3: (Continue)

\begin{tabular}{|c|c|c|c|c|c|c|}
\hline \multirow{2}{*}{ Groups } & \multirow{2}{*}{$\mathbf{n}$} & \multicolumn{2}{|c|}{ PNSW (\%) } & \multicolumn{2}{|c|}{ WRA } & \multirow{2}{*}{ Power ratio } \\
\hline & & Preprandial & Postprandial & Preprandial & Postprandial & \\
\hline Duodenitis & 14 & $57.84 \pm 5.51$ & $65.46 \pm 14.56$ & $61.64 \pm 23.05$ & $143.14 \pm 29.44^{* *}$ & $3.31 \pm 1.49$ \\
\hline Superficial gastritis & 13 & $59.62 \pm 8.21$ & $65.82 \pm 10.63$ & $61.38 \pm 18.35$ & $137.77 \pm 36.76^{* *}$ & $3.98 \pm 1.74^{* *}$ \\
\hline $\begin{array}{l}\text { Chronic superficial } \\
\text { gastritis }\end{array}$ & 23 & $59.58 \pm 6.94$ & $64.8 \pm 7.65$ & $46.78 \pm 25.65$ & $104.45 \pm 53.64^{\Delta}$ & $2.73 \pm 1.6 \# \cdot$ \\
\hline Reflux esophagitis & 16 & $60.29 \pm 7.14^{\star}$ & $68.46 \pm 10.43$ & $61.75 \pm 17.51^{\star}$ & $142.19 \pm 41.38^{* * \star \star} \ldots$ & $3.13 \pm 1.86=$ \\
\hline Functional dyspepsia & 23 & $59.44 \pm 9.52$ & $65.98 \pm 8.05$ & $63.87 \pm 26.89^{\star}$ & $71.46 \pm 32.21^{\# \Delta \Delta}$ & $1.49 \pm 0.91^{* \# \Delta \Delta}$ \\
\hline Normal & 10 & $58.75 \pm 5.46$ & $63.17 \pm 8.29$ & $56.1 \pm 9.08$ & $90 \pm 22.02$ & $2.27 \pm 0.76$ \\
\hline
\end{tabular}

${ }^{*} \mathrm{P}<0.05,{ }^{* *} \mathrm{P}<0.01$ compared with normal group; ${ }^{\#} \mathrm{P}<0.05,{ }^{\# \#} \mathrm{P}<0.01$ compared with superficial gastritis group; ${ }^{\Delta} \mathrm{P}<0.05,{ }^{\Delta \Delta} \mathrm{P}<0.01$ compared with duodentis group; $-\mathrm{P}<0.05$ compared with functional dyspepsia group; ${ }^{\star} \mathrm{P}<0.05,{ }^{\star} \mathrm{P}<0.01$ compared with chronic superficial gastritis group 
Table 4: DP, DF, PNSW, WRA and power ratio of EGG (Channel 2) in patients.

\begin{tabular}{|c|c|c|c|c|c|}
\hline \multirow{2}{*}{ Groups } & \multirow{2}{*}{$\mathrm{n}$} & \multicolumn{2}{|c|}{$\mathrm{DP}(\mu \mathrm{V})$} & \multicolumn{2}{|c|}{ DF(cpm) } \\
\hline & & Preprandial & Postprandial & Preprandial & Postprandial \\
\hline Duodenitis & 14 & $186.14 \pm 75.22$ & $343.5 \pm 91.89^{*}$ & $3.18 \pm 0.25$ & $3.46 \pm 0.37$ \\
\hline Superficial gastritis & 13 & $210.85 \pm 90.36$ & $345.31 \pm 73.27^{* *}$ & $3.3 \pm 0.31$ & $3.29 \pm 0.34$ \\
\hline Chronic superficial gastritis & 23 & $138.61 \pm 63.89^{\# \# \Delta}$ & $202.61 \pm 84.84^{* \# \# \Delta \Delta}$ & $3.27 \pm 0.27^{*}$ & $3.2 \pm 0.28$ \\
\hline Reflux esophagitis & 16 & $167.5 \pm 51.96$ & $317.94 \pm 89.84=$ & $3.39 \pm 0.27^{* *}$ & $3.28 \pm 0.25$ \\
\hline Functional dyspepsia & 23 & $175.48 \pm 91.06$ & $191.65 \pm 95.93^{* \# \# \Delta \Delta}$ & $3.36 \pm 0.33^{*}$ & $3.31 \pm 0.27$ \\
\hline Normal & 10 & $175.2 \pm 42.98$ & $263.3 \pm 58.42$ & $3.05 \pm 0.28$ & $3.2 \pm 0.33$ \\
\hline
\end{tabular}

Table 4: (Continue)

\begin{tabular}{|l|c|c|c|c|c|c|}
\hline \multirow{2}{*}{\multicolumn{1}{c|}{ Groups }} & \multirow{2}{*}{$\mathbf{n}$} & \multicolumn{2}{c|}{ PNSW (\%) } & \multicolumn{2}{c|}{ WRA } & \multirow{2}{*}{ power ratio } \\
\cline { 3 - 7 } & & Preprandial & Postprandial & Preprandial & Postprandial & \\
\hline Duodenitis & 14 & $60.21 \pm 8.02$ & $62.63 \pm 10.69$ & $66.07 \pm 23.63$ & $122.57 \pm 32.18^{*}$ & $2.99 \pm 1.75^{*}$ \\
\hline Superficial gastritis & 13 & $64.83 \pm 11.06$ & $64.75 \pm 10.94$ & $75 \pm 29.17$ & $122.54 \pm 26.88^{*}$ & $2.47 \pm 1.92$ \\
\hline Chronic superficial gastritis & 23 & $61.53 \pm 8.95$ & $64.59 \pm 6.87$ & $50.57 \pm 23.18^{\# \#}$ & $70.87 \pm 27.28^{* * \# \Delta \Delta}$ & $2.09 \pm 1.65$ \\
\hline Reflux esophagitis & 16 & $58.24 \pm 7.06$ & $65.68 \pm 10.24$ & $60.56 \pm 19.51$ & $109.19 \pm 31.68 *$ & $2.64 \pm 1.28^{*}=$ \\
\hline Functional dyspepsia & 23 & $61.25 \pm 8.11$ & $64.48 \pm 10.85$ & $61.57 \pm 29.76$ & $66.61 \pm 28.77^{* * \# \Delta \Delta}$ & $1.58 \pm 1.57^{\Delta}$ \\
\hline Normal & 10 & $64.17 \pm 6.93$ & $63.27 \pm 7.61$ & $63.7 \pm 10.83$ & $98.3 \pm 21.28$ & $1.72 \pm 0.3$ \\
\hline
\end{tabular}

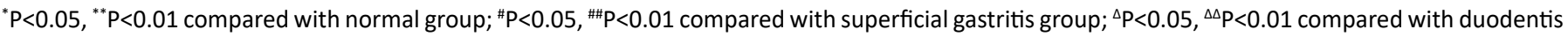
group; $\cdot P<0.05$ compared with functional dyspepsia group; ${ }^{\star} P<0.05, \star \star P<0.01$ compared with chronic superficial gastritis group

Table 5: DP, DF, PNSW, WRA and power ratio of EGG (Channel 3) in patients.

\begin{tabular}{|c|c|c|c|c|c|}
\hline \multirow{2}{*}{ Groups } & \multirow{2}{*}{$\mathbf{n}$} & \multicolumn{2}{|c|}{$\mathrm{DP}(\mu \mathrm{V})$} & \multicolumn{2}{|c|}{ DF(cpm) } \\
\hline & & Preprandial & Postprandial & Preprandial & Postprandial \\
\hline Duodenitis & 14 & $165.86 \pm 69.19$ & $419 \pm 128.12^{* \star \star \star}$ & $3.4 \pm 0.36$ & $3.32 \pm 0.38$ \\
\hline Superficial gastritis & 13 & $220.23 \pm 98.02$ & $412.62 \pm 97.65^{* *}$ & $3.39 \pm 0.44$ & $3.1 \pm 0.19^{*}$ \\
\hline Chronic superficial gastritis & 23 & $142.61 \pm 65.99^{\# \#}$ & $271.78 \pm 136.56^{\# \#}$ & $3.25 \pm 0.29$ & $3.21 \pm 0.28$ \\
\hline Reflux esophagitis & 16 & $202.44 \pm 69.12^{\star \star}$ & $408.75 \pm 112.72^{* *}$ & $3.21 \pm 0.40$ & $3.23 \pm 0.26$ \\
\hline Functional dyspepsia & 23 & $185.61 \pm 73.46^{\star}$ & $262 \pm 151.37^{\# \#}$ & $3.32 \pm 0.26$ & $3.31 \pm 0.39$ \\
\hline Normal & 10 & $182.4 \pm 27.05$ & $271.7 \pm 43.25$ & $3.2 \pm 0.21$ & $3.3 \pm 0.19$ \\
\hline
\end{tabular}

Table 5: (Continue)

\begin{tabular}{|l|c|c|c|c|c|c|}
\hline \multirow{2}{*}{\multicolumn{1}{c|}{ Groups }} & \multirow{2}{*}{$\mathbf{n}$} & \multicolumn{2}{c|}{ PNSW (\%) } & \multicolumn{2}{c|}{ WRA } & \multirow{2}{*}{ power ratio } \\
\cline { 3 - 7 } & & Preprandial & Postprandial & Preprandial & Postprandial & \\
\hline Duodenitis & 14 & $57.01 \pm 6.65$ & $65.45 \pm 11.38$ & $60.57 \pm 22.26$ & $145.14 \pm 37.20^{* \star \star \star}$ & $4.22 \pm 2.29^{* *}$ \\
\hline Superficial gastritis & 13 & $63.15 \pm 10.19$ & $67.7 \pm 10.64$ & $79.77 \pm 29.44$ & $140.38 \pm 30.43^{* *}$ & $2.69 \pm 2.02$ \\
\hline Chronic superficial gastritis & 23 & $60.18 \pm 8.58$ & $67.24 \pm 8.83$ & $51.52 \pm 24.04^{\# \#}$ & $92.48 \pm 44.93^{\# \#}$ & $3.27 \pm 2.37$ \\
\hline Reflux esophagitis & 16 & $60.13 \pm 6.94$ & $70.83 \pm 8.48^{*}$ & $71.25 \pm 21.37^{\star}$ & $137.19 \pm 33.94^{* *}$ & $3.28 \pm 1.56^{* *}$ \\
\hline Functional dyspepsia & 23 & $59.22 \pm 6.92$ & $67.45 \pm 9.91$ & $65.87 \pm 21.71^{\star}$ & $88.70 \pm 50.06^{\# \#}$ & $2.10 \pm 2.04$ \\
\hline Normal & 10 & $61.04 \pm 6.86$ & $63.57 \pm 7.80$ & $65.3 \pm 8.94$ & $99.6 \pm 14.91$ & $1.83 \pm 0.37$ \\
\hline
\end{tabular}

${ }^{*} \mathrm{P}<0.05,{ }^{* *} \mathrm{P}<0.01$ compared with normal group; ${ }^{\# P}<0.05$, ${ }^{\# \#} \mathrm{P}<0.01$ compared with superficial gastritis group; ${ }^{\triangle} \mathrm{P}<0.05,{ }^{\Delta \Delta} \mathrm{P}<0.01$ compared with duodentis group; $\cdot P<0.05$ compared with functional dyspepsia group; ${ }^{\star} P<0.05,{ }^{\star}{ }^{*} P<0.01$ compared with chronic superficial gastritis group 


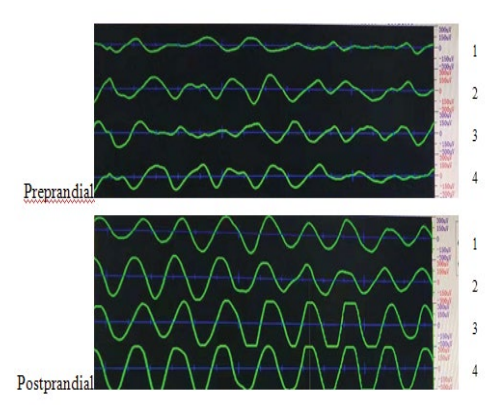

Superficial gastritis
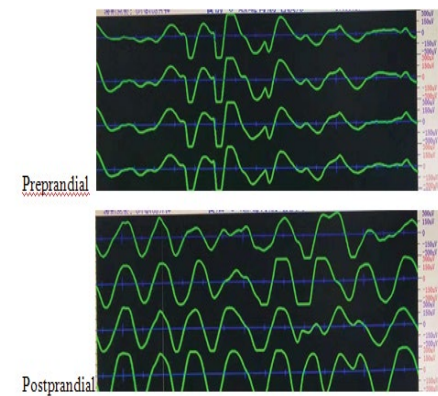

Reflux esophagitis

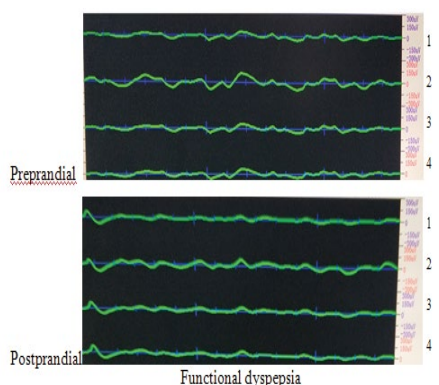

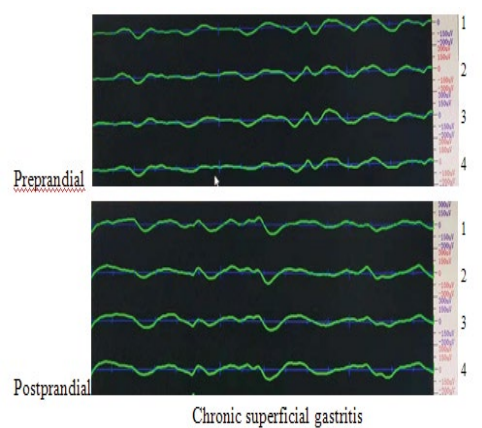

Chronic superficial gastritis

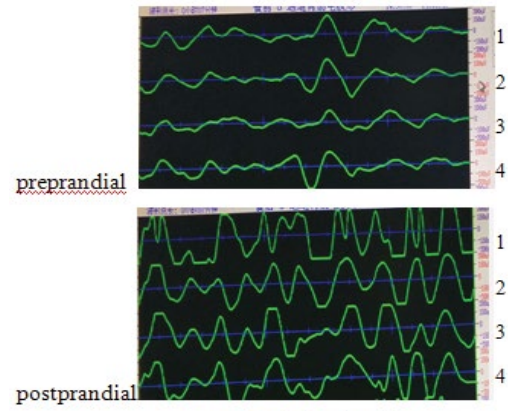

Gastric duodenitis

Electrode 1: Gastric body

2: Gastric lesser curvature

3: Gastric larger curvature

4: Gastric antrum

Figure 1: Raw signal of the EGG in patients with CAP.

\section{Disclaimer}

None

\section{Conflict of Interest}

None

\section{Funding Sources}

None

\section{References}

1. Perquin CW, Hazebroek-Kampschreur AA, Hunfeld JA, van SuijlekomSmit LW, Passchier J, et al. (2000) Chronic pain among children and adolescents: physician consultation and medication use. Clin J Pain 16: 229-235.

2. Gu XB, Huang $X Q$, Wang $C X$ (2011) Diagnostic value of gastroenterogram in children with abdominal pain. Clin Rational Drug Use 4: 82-84.

3. Kayar Y, Danalıoğlu A, Kafee AA, Okkesim Ş, Şentürk H (2016) Gastric myoelectrical activity abnormalities of electrogastrography in patients with functional dyspepsia. Turk J Gastroenterol 27: 415420.
4. Komorowski D (2018) EGG DWPack: System for Multi-Channel Electrogastrographic Signals Recording and Analysis. J Med Syst 42: 201.

5. Ke MY (2000) Summary of the national electrogastrogram academic conference of the Chinese medical association. Chin J Internal Med 39: 202-203.

6. Meng SH, Ji HJ, Li ZJ, Wei XH, Jin YJ, et al. (2014) Diagnosis and treatment of 280 cases of acute abdominal pain. Northern Pharmaceutical 10: 126.

7. Guo LM, Huang XQ, Wang CX (2013) Application analysis of electrogastrogram in clinical diagnosis and treatment of anorexia in children. Chin J Medical Guide 10: 302.

8. Chen YL (2011) Clinical significance of gastroscopy in children with recurrent abdominal pain. China Health Industry 8: 104.

9. Ma G, Dai WJ (2013) Analysis of electrogastrogram and gastric emptying in patients with functional dyspepsia. Chinese J postgraduates Medicine 36: 34-36.

10. Uścinowicz M, Jarocka-Cyrta E, Kaczmarski M (2005) Electrogastrography in children with functional abdominal pain and gastritis. Pol Merkur Lekarski 18: 54-57. 